García, J.A.; Cárdenas, A.; Burgos, S.; Santiago, C.; Hernández, F.; Sanz, V.; Fernandez-delValle, M.; Rubio, M. y Pérez, M. (2019). Estilo de vida y distribución de grasa en adolescentes asmáticos y sanos / Lifestyle and Fat Distribution in Asthmatic and Healthy Adolescents. Revista Internacional de Medicina y Ciencias de la Actividad Física y el Deporte vol. 19 (73) pp. 107-118 Http://cdeporte.rediris.es/revista/revista73/artefectos999.htm

DOI: http://doi.org/10.15366/rimcafd2019.73.008

\title{
ORIGINAL
}

\section{ESTILO DE VIDA Y DISTRIBUCIÓN DE GRASA EN ADOLESCENTES ASMÁTICOS Y SANOS}

\section{LIFESTYLE AND FAT DISTRIBUTION IN ASTHMATIC AND HEALTHY ADOLESCENTS}

\author{
García, J.A.'; Cárdenas, A. ${ }^{1}$; Burgos, S. ${ }^{2}$; Santiago, C. ${ }^{2}$; Hernández, F. ${ }^{3}$; \\ Sanz, V.4; Fernandez-del-Valle, M. ${ }^{5}$; Rubio, M. ${ }^{6}$ y Pérez, M. ${ }^{6}$ \\ ${ }^{1}$ Máster universitario oficial Actividad Física y Salud. Universidad Europea de Madrid (España) \\ Joseangelgarciamerino@gmail.com, caan.ligo@gmail.com \\ 2 Profesoras titulares de Universidad. Universidad Europea de Madrid (España) \\ Silvia.burgos@universidadeuropea.es, catalina.santiago@universidadeuropea.es \\ ${ }^{3}$ Grado en Ciencias de la Actividad Física y Deporte. Universidad Europea de Madrid (España) \\ fhm2026@hotmail.com \\ 4 Licenciada en Medicina y Cirugía. Especialista en Neumología Pediátrica. Hospital Ramón y \\ Cajal (España) veross81@yahoo.es \\ ${ }^{5}$ Assistant Professor, Department of Applied Health. Southern Illinois University Edwardsville \\ (US) marfern@siue.edu \\ 6 Catedráticas. Universidad Europea de Madrid (España)
} margarita.rubio@universidadeuropea.es, margarita.perez@universidadeuropea.es

\section{AGRADECIMIENTOS Y/O FINANCIACIÓN}

Financiado a través de la VII Convocatoria Real Madrid- Universidad Europea (Ref 2015/03RM); Tercer premio en los XV Premios Neumomadrid; Beca Jóvenes Investigadores de la SENP 2015.

Código UNESCO / UNESCO code: 3201.10 Ciencia Clínica Pediátrica / Clinical Science Pediatric

Clasificación del Consejo de Europa / Council of Europe classification: 11. Medicina Deportiva / Sports Medicine

Recibido 3 de abril de 2017 Received April 3, 2017

Aceptado 16 de diciembre de 2017 Accepted December 16, 2017

\section{RESUMEN}

Introducción: el objetivo es analizar el estilo de vida, en función del ejercicio y la dieta, de un grupo de adolescentes de la Comunidad de Madrid, 
teniendo en cuenta género y patología y su relación con el estado nutricional, la distribución de grasa y la función pulmonar. Metodología: Estudio descriptivo de 207 sujetos que analizó el estilo de vida a partir del nivel de actividad física (AF) y la adherencia a la dieta mediterránea, el estado nutricional y la distribución de grasa, y la salud respiratoria. Resultados: El grupo de no asmáticos fue más activo $(p=0,003)$ y presentó menor ICT $(p=0,001)$ que el grupo de asmáticos. Se encontraron diferencias significativas dentro del grupo sin asma en nivel de AF siendo los varones más activos $(\mathrm{p}=0,01)$ y presentando menor índice cinturatalla (ICT) que las mujeres del mismo grupo $(p=0,001)$. Conclusiones: Los adolescentes no asmáticos fueron más activos y presentaron mejor distribución de grasa que los asmáticos.

PALABRAS CLAVE: asma, adolescentes, ejercicio físico, dieta mediterránea

\section{ABSTRACT}

Objectives: objective is to analyze the lifestyle, integrating exercise and diet, of a group of adolescents from the Community of Madrid, taking into account gender and pathology and its relationship with nutritional status, fat distribution and lung function in adolescents with or without asthma. Methods: This was a descriptive study including 207 subjects aged $13.20 \pm 0.62$ years. Lifestyle was assessed in terms of physical activity (PA) levels, Mediterranean diet, nutritional status, and respiratory health measured through $\mathrm{FEV}_{1}(\mathrm{z})$. Results: In the nonasthma group, boys were more active $(p=0.01)$ and showed a lower waist-toheight ratio $(\mathrm{WHtR})$ than girls $(p=0.001)$. Participants without asthma were more active $(p=0.003)$, and had a better WHtR $(p=0.001)$ and FEV $1(p=$ 0.001 ) than those with asthma. Conclusions: In this Spanish population sample, non-asthmatic adolescents were more active and showed a better nutritional status, fat distribution and respiratory health than their peers with asthma.

KEYWORDS: asthma, adolescents, physical exercise, Mediterranean diet

\section{INTRODUCCIÓN}

Estudios epidemiológicos alertan de la tendencia a la inactividad física en todo el mundo y predicen que los niños y adolescentes de hoy son la primera generación que tiene una expectativa de vida menor que la de sus padres ${ }^{1}$. La inactividad física es el cuarto factor de riesgo de mortalidad en la población actual dentro del mundo desarrollado y causa el $6 \%$ de las defunciones a nivel mundial. España es uno de los países del mundo más afectados por la epidemia de la inactividad física, lo que aumenta el riesgo de enfermedad cardiovascular, diabetes, obesidad y síndrome metabólico, descendiendo así la expectativa de vida ${ }^{2}$.

El asma es una enfermedad inflamatoria crónica de las vías respiratorias, en cuya patogenia intervienen diversas células y mediadores de la inflamación, condicionada en parte por factores genéticos y ambientales y que cursa con 
hiper-respuesta bronquial y obstrucción al flujo aéreo ${ }^{3}$. Se caracteriza por síntomas como, ataques recurrentes de disnea, sibilancias, opresión en el pecho y/o tos. Asimismo, los síntomas experimentados por parte de la población infantil y adolescente que padece esta enfermedad pueden verse agudizados con la AF, desencadenando temor frente a la realización de la misma y traduciéndose en un deterioro del estado de salud general que limita el desempeño en las actividades cotidianas ${ }^{4}$. El comportamiento sedentario que se asocia con los adolescentes que padecen asma puede empeorar la evolución de la enfermedad y facilitar la aparición de sobrepeso y obesidad ${ }^{5}$.

La Organización Mundial de la Salud (OMS) calcula que en la actualidad hay 235 millones de pacientes con asma ocasionando 18.000 muertes anuales $^{6}$. La prevalencia de padecer la enfermedad es creciente en países desarrollados destacando las áreas urbanas donde los adolescentes son menos activos, más obesos y presentan una menor adherencia a la dieta mediterránea ${ }^{7}$. El coste total del asma en pediatría en España es de aproximadamente 532 millones de euros, oscilando entre 392 y 693 millones de euros. El coste medio anual por paciente asmático es de 1.149 euros, y varía entre 403 euros para la categoría leve y 5.380 euros para la más grave ${ }^{8}$.

La causa del aumento en la prevalencia y gravedad del asma en la última década parece ser multifactorial. Se encuentran factores como la exposición a alérgenos 0 agentes irritantes, cambios de temperatura, infecciones respiratorias virales y predisposición genética ${ }^{6}$. Sin embargo, cada vez hay más evidencia científica que muestra el efecto que tienen factores ambientales tales como el ejercicio y la dieta mediterránea en la población asmática, y su relación directa con la obesidad ${ }^{9}$ y el grado de adiposidad visceral ${ }^{10}$ agravando la sintomatología. El estudio Physical Activity, Nutrition, and Allergies in Children Examined in Athens (PANACEA) muestra que la dieta mediterránea tiene un efecto protector sobre los síntomas del asma, la inflamación de la vía aérea y la función pulmonar ${ }^{11}$. Pocos estudios diagnostican previamente el estilo de vida de la población y analizan de forma integrada el efecto de la calidad de la dieta utilizada y el ejercicio físico realizado sobre variables de estado nutricional.

Por otro lado, padecer asma puede convertirse en un factor de riesgo para el desarrollo de un estado nutricional inadecuado pudiendo promover el sobrepeso o la obesidad, ya que la población adolescente actual está reduciendo su participación en actividades físicas tanto dentro como fuera del ámbito escolar, aumentando el comportamiento sedentario y dedicando más tiempo a estar frente a la pantalla del televisor o a utilizar vídeo juegos en su tiempo libre ${ }^{4,12}$. Confirmando así la relación entre el menor nivel de actividad física y la mayor prevalencia de la enfermedad ${ }^{13}$.

La AF tiene un importante papel sobre el asma al mantener un mejor control del índice de masa corporal (IMC), y disminuir el porcentaje de grasa visceral lo que condiciona un menor nivel inflamatorio sistémico mostrando un descenso de la lgE. Todo ello, podría repercutir positivamente en la mejora de la calidad de vida del adolescente asmático ${ }^{14}$. Los pacientes con asma pueden mostrar menor tolerancia al ejercicio debido al empeoramiento de los síntomas de la 
enfermedad durante el ejercicio, o a otros motivos como la falta de condición física debido a la inactividad.

Por consiguiente, el presente estudio tiene como objetivo analizar el estilo de vida en función del ejercicio y la dieta de un grupo de adolescentes de la Comunidad de Madrid, teniendo en cuenta género y patología y su relación con el estado nutricional, la distribución de grasa y la función pulmonar.

\section{MATERIAL Y MÉTODO}

Para la realización de este estudio se utilizó un diseño descriptivo observacional. El estudio fue aprobado por el Comité Ético de Investigación Clínica del Hospital Infantil Universitario Niño Jesús de Madrid (№ referencia: R0031/14).

\section{Sujetos}

Se analizó el estilo de vida, el estado nutricional y el patrón respiratorio de 207 sujetos (132 varones) con edades entre los 12 y 14 años y características antropométricas homogéneas (edad, peso y talla). De ellos, 60 eran pacientes asmáticos (40 varones) que acudieron a la consulta de neumología del Hospital Infantil Universitario Niño Jesús de Madrid entre octubre de 2015 y junio de 2016; y 147 adolescentes (92 varones) de primero y segundo de secundaria del Eurocolegio Casvi de Boadilla del Monte, sin presencia de síntomas asmáticos, analizado a través del cuestionario Internacional Study of Asthma and Allergies in Childhood (ISAAC) y valorados entre febrero y marzo de 2016.

\section{Variables}

\section{Estilo de vida: Calidad de la dieta mediterránea}

Para evaluar la calidad de la dieta mediterránea de los sujetos del estudio se utilizó el test de calidad de la dieta mediterránea KidMed. El cuestionario consta de 16 items que deben contestarse como verdadero o falso, de los cuales 12 representan hábitos saludables que, de cumplirse, puntúan positivamente $(+1)$; y 4 que implican costumbres incorrectas y que puntúan negativamente $(-1)$. La puntuación total menor a 3 indica baja calidad, entre 4 y 7 calidad media y mayor a 8 calidad óptima de la dieta mediterránea ${ }^{15}$.

\section{Estilo de vida: Nivel de Actividad Física}

Se utilizó el cuestionario PAQ-C (Physical Activity Questionnaire for Children) que evalúa el nivel de actividad física realizado en los últimos 7 días en niños y adolescentes de entre 7 y 14 años, ambos incluidos. Consiste en 10 items, 9 de los cuales se utilizan para medir el nivel de actividad física y el último evalúa si una enfermedad u otro acontecimiento impidió que el niño o adolescente hiciera sus actividades regulares. 
El resultado global es una puntuación de 1 a 5 . Cuando la puntuación alcanzada es menor a 2,33 indica bajo nivel de actividad física; cuando está entre 2,33 y 3,66 indica moderado nivel de actividad física y cuando es mayor de 3,66 indica que es activo físicamente. Se excluyeron del análisis todos aquellos niños y adolescentes que respondían "Sí" a la pregunta "Estuvo enfermo en la última semana"16.

\section{Estado nutricional y distribución de grasa}

Se midió peso corporal y altura a través de una báscula mecánica ASIMED modelo BARYS PLUS C con tallímetro telescópico, para el cálculo del Índice de Masa Corporal (IMC), considerando los valores de corte sugeridos para población de entre 5 y 19 años según la Organización Mundial de la Salud convertidos a puntuación Z-score, donde se permite estandarizar el estado nutricional sin que afecten variables relevantes como el sexo, edad y raza, obtenida a través del enlace web: http://www.who.int/growthref/tools/en/. Se establece la siguiente categorización: Obesidad: $\geq+2 \mathrm{DE}$; Sobrepeso: >+1DE; Normopeso: entre -1 y +1DE; Delgadez: $\leq-2 D E$; Delgadez grave: $\leq-3 D E^{17}$.

El índice cintura talla (ICT) relacionado con el porcentaje de grasa visceral intra-abdominal, se midió a partir del perímetro de cintura utilizando una cinta métrica KAWE 1,5 × $8 \mathrm{~mm}$. Se tomó el punto equidistante entre la última costilla no flotante y la cresta iliaca ${ }^{18}$, y se dividió por la talla en centímetros. En edad pediátrica se establecen los siguientes valores de referencia: distribución normal de grasa visceral $\leq 0,47$; distribución moderada de grasa visceral entre 0,47 y 0,50 ; exceso de grasa visceral $>0,50^{19}$.

\section{Salud respiratoria}

Se utilizó el cuestionario ISAAC para detectar síntomas asociados al asma en los sujetos del Eurocolegio Casvi. El cuestionario que se utiliza como screening fue contestado de manera individual por los adolescentes y bajo la supervisión de los investigadores, siguiendo la metodología utilizada en estudios internacionales anteriores de prevalencia de síntomas de asma en escolares y adolescentes ${ }^{20}$.

El patrón respiratorio se midió con un espirómetro modelo Spirostik, software Blue Cherry, de Geratherm®. Las variables obtenidas fueron Volumen Espiratorio forzado en el primer segundo (FEV1 o VEMS), puede expresarse en valor absoluto o porcentual (FEV1\%). Capacidad Vital Forzada (FVC). Flujo espiratorio medio (FEF25-75\% o MMEF). Los datos obtenidos fueron evaluados siguiendo las recomendaciones que valoran las ecuaciones espirométricas de referencia recogidas en la Global Lung Initiative (GLI) de 2012 21 , como nuevo paradigma a la hora de evaluar las espirometrías, y que establece como límite inferior de normalidad (LIN) un valor Z-score en FEV 1 para población sana comprendido entre -1,96 y 1,96; y para población con síntomas de patología respiratoria entre $-1,64$ y $1,64^{22}$. 


\section{Análisis estadístico}

Las frecuencias de variables cualitativas se expresaron como número absoluto y porcentaje. Las variables cuantitativas analizadas fueron expresadas como media \pm desviación típica (DT). Para el análisis de asociación entre variables cualitativas se utilizó la prueba $X^{2}$ de Pearson. La comparación entre las medias de las variables cuantitativas fue evaluada usando la $t$-Student para muestras independientes después de comprobar que la variable seguía una distribución normal mediante el test de Kolmogorov-Smirnov. Se consideraron estadísticamente significativos valores de $p$ inferiores a 0,05.

Para el análisis de datos se utilizó el paquete estadístico SPSS versión 19.0 (Chicago, IL, USA).

\section{RESULTADOS}

\section{Características de los sujetos}

Se analizaron un total de 207 sujetos (132 varones), de entre 12 y 14 años. Las características de la muestra se detallan en la Tabla 1.

\begin{tabular}{c|cc|cc} 
& \multicolumn{5}{c}{ Tabla 1. Características de los sujetos } \\
& \multicolumn{2}{c}{ Asmáticos $(n=60)$} & No asmáticos $(n=147)$ \\
\hline & Varones & Mujeres & Varones & Mujeres \\
& $(n=40)$ & $(n=20)$ & $(n=92)$ & $(n=55)$ \\
& $M \pm D T$ & $M \pm D T$ & $M \pm D T$ & $M \pm D T$ \\
Edad & $13,13 \pm 0,56$ & $12,96 \pm 0,57$ & $13,06 \pm 0,53$ & $13,08 \pm 0,45$ \\
Peso & $51,53 \pm 11,94$ & $53,30 \pm 17,02$ & $49,24 \pm 10,81$ & $49,15 \pm 9,25$ \\
Talla & $1,56 \pm 0,08$ & $1,54 \pm 0,09$ & $1,58 \pm 0,09$ & $1,59 \pm 0,07$ \\
\multicolumn{4}{c}{ Edad (años); Peso (kilogramos); Talla (metros) }
\end{tabular}

\section{Estilo de vida y estado nutricional según género y patología}

A continuación, se muestran las variables analizadas según el sexo en cada uno de los grupos estudiados (Tabla 2). Se observan diferencias estadísticamente significativas dentro del grupo de sujetos sin asma, donde los varones realizan mayor nivel de AF que las mujeres $(p=0,01)$. A su vez, los varones sin asma presentan un ICT más favorable que las mujeres que es estadísticamente significativo $(p=0,001)$.

Los resultados de la comparación entre sujetos asmáticos y no asmáticos en relación a las variables analizadas se presentan en la Tabla 3. Encontramos diferencias significativas a favor del grupo sin asma, los cuales realizan un mayor nivel de actividad física frente a los asmáticos $(p=0,003)$. A su vez, el grupo sin asma presenta de manera significativa un menor ICT $(p=0,001)$ (tabla 3$)$. Además, no se encontraron cambios en el ICT en ninguna de las categorías establecidas para el nivel de AF (baja, moderada o alta actividad física) en ambos 
grupos, asmáticos o sanos, tal como se representa en la Figura 1. Asimismo, existen diferencias estadísticamente significativas en $\mathrm{FEV}_{1}(\mathrm{Z})$ a favor del grupo no asma $(p=0,001)$.

Tabla 2. Estilo de vida, estado nutricional y salud respiratoria según género y patología

\begin{tabular}{|c|c|c|c|c|c|c|}
\hline & \multicolumn{3}{|c|}{ Asmáticos $(\mathrm{n}=60)$} & \multicolumn{3}{|c|}{ No Asmáticos $(n=147)$} \\
\hline & $\begin{array}{c}\text { Varones } \\
(n=40) \\
M \pm D T\end{array}$ & $\begin{array}{c}\text { Mujeres } \\
(n=20) \\
M \pm D T\end{array}$ & $P$ & $\begin{array}{c}\text { Varones } \\
(\mathrm{n}=92) \\
\mathrm{M} \pm \mathrm{DT}\end{array}$ & $\begin{array}{c}\text { Mujeres } \\
(n=55) \\
M \pm D T\end{array}$ & $P$ \\
\hline KIDMED & $6,78 \pm 1,84$ & $6,70 \pm 1,89$ & 0,88 & $7,16 \pm 2,05$ & $6,82 \pm 2,13$ & 0,33 \\
\hline PAQ-C & $2,59 \pm 0,65$ & $2,31 \pm 0,59$ & 0,20 & $3,00 \pm 0,79$ & $2,67 \pm 0,63$ & 0,01 \\
\hline $\begin{array}{c}\text { IMC/Edad } \\
(Z)\end{array}$ & $0,46 \pm 1,60$ & $0,58 \pm 1,49$ & 0,79 & $0,10 \pm 1,09$ & $-0,13 \pm 0,90$ & 0,18 \\
\hline $\mathrm{ICT}$ & $0,48 \pm 0,08$ & $0,46 \pm 0,57$ & 0,40 & $0,41 \pm 0,39$ & $0,44 \pm 0,05$ & 0,001 \\
\hline $\mathrm{FEV}_{1}(\mathrm{Z})$ & $-0,83 \pm 1,34$ & $-1,34 \pm 1,08$ & 0,14 & $0,89 \pm 1,14$ & $0,89 \pm 1,10$ & 0,98 \\
\hline
\end{tabular}

KIDMED: Índice de calidad de la dieta mediterránea en niños y adolescentes; PAQ-C: Physical Activity Questionnaire for Children; IMC/Edad (Z): Índice de Masa Corporal categorizado por edades y Z-score; ICT: Índice Cintura-Talla; FEV ${ }_{1}(Z)$ : volumen espirado en el primer segundo categorizado por Z-score.

Tabla 3. Estilo de vida, estado nutricional y salud respiratoria según patología

\begin{tabular}{c|c|cc} 
& Asmáticos $(\mathrm{n}=60)$ & No Asmáticos $(\mathrm{n}=147)$ & \\
\hline & $\mathrm{M} \pm \mathrm{DT}$ & $\mathrm{M} \pm \mathrm{DT}$ & $P$ \\
KIDMED & $6,75 \pm 1,84$ & $7,03 \pm 2,08$ & 0,36 \\
PAQ-C & $2,48 \pm 0,63$ & $2,88 \pm 0,75$ & 0,003 \\
IMC/Edad $(\mathrm{Z})$ & $0,50 \pm 1,55$ & $0,01 \pm 1,02$ & 0,03 \\
ICT & $0,47 \pm 0,08$ & $0,43 \pm 0,04$ & 0,001 \\
FEV $_{1}(\mathrm{Z})$ & $-1,00 \pm 1,27$ & $0,89 \pm 1,12$ & 0,001
\end{tabular}




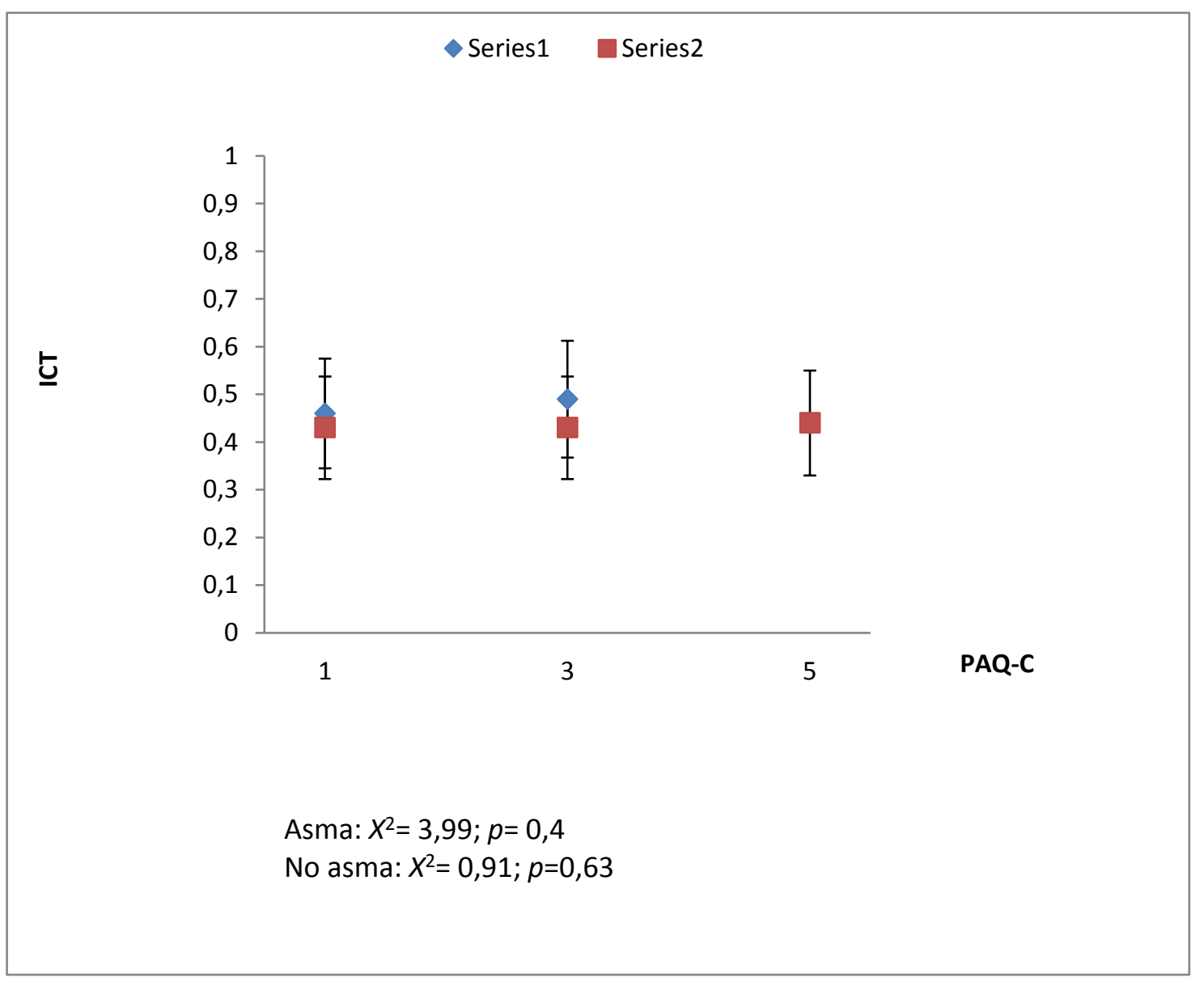

Figura 1. Relación entre nivel de AF e ICT para grupo de adolescentes asmáticos y sanos

\section{DISCUSIÓN}

La puntuación media obtenida del cuestionario PAQ-C determina que los sujetos tienen un moderado nivel de actividad física, encontrando diferencias significativas entre sexos dentro del grupo sin asma, donde los varones son más activos que las mujeres. Estos datos se correlacionan con el estudio de Corder et al. ${ }^{23}$, en el que los adolescentes están reemplazando diez minutos de AF al día por actividades sedentarias. Basándonos en investigaciones previas, se oberva que el aumento en actividades sedentarias puede estar relacionado con más tiempo dedicado a ver televisión, especialmente las niñas ${ }^{24}$. Este aumento en tiempo sedentario ha sido vinculado a la reducción significativa de los niveles de actividad física, siendo mas pronunciado en mujeres. Estas diferencias en niveles de actividad física en función del sexo también han sido observadas en niños y adolescentes de otros países europeos ${ }^{25}$, aumentando el riesgo de padecer sobrepeso y obesidad, y desarrollar enfermedades cardio-metabólicas en la edad adulta. Es por ello, que se recomienda el dessarollo de programas de promoción para aumentar el nivel de actividad física, con especial atención en las niñas ${ }^{26}$. Ademas de la reducción evidente de los niveles de actividad física, los valores obenidos en el cuestionario KidMed confirman la necesidad de mejorar también sus habitos alimentarios hacia un modelo más próximo a la dieta mediterránea. La tendencia de los paises mediterráneos a abandonar la dieta 
mediterránea se ha incrementado en los últimos años, especialmente en niños y adolescentes ${ }^{27}$. Así mismo, en línea con nuestro estudio, los datos obtenidos en el estudio PANACEA ${ }^{11}$, ponen en relieve la necesidad de apostar por políticas que mejoren la educación y los hábitos nutricionales. Finalmente, se ha vinculado baja adherencia a la dieta mediterránea con bajos niveles de actividad física tal y como apuntan los resultados de nuestro estudio ${ }^{25}$.

Comparando el estilo de vida entre grupos, observamos que los adolescentes que no tienen asma son más activos y presentan menor ICT. Datos que se relacionan con los reportados por Walders-Abramson et al..$^{5}$, donde concluyen que los adolescentes con asma tienen una probabilidad del $21 \%$ de padecer sobrepeso y ser clasificados como obesos en edad adulta por su bajo nivel de AF. Niveles altos de actividad física parece que pueden prevenir la evolución negativa de la enfermedad, y el ejercicio físico puede reducir síntomas debido a reducciones en los niveles de inflamación, mejoras de la capacidad pulmonar, y mejora de la condición de los músculos respiratorios ${ }^{12}$. En cuanto a salud respiratoria se observó que en el grupo de sujetos no asmáticos, a mayor nivel de AF y adherencia óptima a la dieta mediterránea, mejor valor de FEV 1 (Z). Asimismo, en el grupo con asma se halló que cuanto menor nivel de actividad física, más afectado se encuentra el valor de $\mathrm{FEV}_{1}(\mathrm{Z})$. Por tanto, el incremento de niveles de AF en la población asmática es esencial para mejorar su estado de salud general y respiratorio ${ }^{5,12,13}$

A pesar de que los datos obtenidos en niveles de actividad física y dieta cumplieron la hipótesis, los resultados en cuanto a la relación entre el cumplimiento de la dieta mediterránea y la función pulmonar no fueron los esperados en el grupo de asmáticos. Una dieta óptima no se relacionó con un mejor valor de $\mathrm{FEV}_{1}(\mathrm{Z})$. Estos datos son similares a los publicados por González et $a .^{28}$, donde no se observó el efecto protector de la dieta mediterránea sobre la salud respiratoria en pacientes asmáticos. Sin embargo, otros estudios sí evidencian la relación inversa entre dieta mediterránea y la prevalencia de los síntomas del asma y mejores valores respiratorios ${ }^{7,9,11}$. No está muy claro como la dieta mediterránea promueve estos cambios positivos en la sintomatología, pero parece ser que la proporción en macronutrientes tiene un efecto positivo en el nivel de estrés oxidativo e inflamación presente en pacientes con asma ${ }^{9}$.

Nuestro estudio tiene algunas limitaciones. Algunos sujetos pueden haber modificado sus habitos de alimentación y ejercicio después del diagnotico médico. Así mismo, al identificar las variables de confusión (edad, peso y talla), el tamaño de la muestra se redujo de tal forma que las conclusiones obtenidas, no pueden ser trasladadas a toda la población adolescente asmática. EI IMC (Z) y el ICT son variables estimativas y pueden verse afectadas por la edad de los sujetos analizados, ya que se encuentran en una etapa de crecimiento donde el predominio del peso puede ser muscular. Finalmente, la utilización de herramientas validadas, pero subjetivas, puede haber provocado pequeñas diferencias en el registro de calidad de la dieta y de actividad física. En este caso, la utilización de herramientas objetivas, como es la utilización de acelerómetros para cuantificar el nivel de AF como refleja Eijkemans et al. ${ }^{29}$ serían más recomendables para un registro más preciso. Asimismo, registrar la composion corporal a tarves de densitometria como se realiza en el trabajo de Rosenkranz 
et al..$^{30}$, proporcionaría datos más objetivos. Sin embargo, todas las herramientas utilizadas en este estudio han sido utilizadas anteriormente en ambas poblaciones (asmática y no asmática), informando de resultados muy similares a los obtenidos en este trabajo.

Hacer este tipo de trabajos que tratan de analizar y hacer un diágnostico del estilo de vida en población adolescente con patología ayudan a indicar de forma más precisa sus necesidades concretas, y por tanto, las intervenciones necesarias para mejorar así su salud en un futuro.

\section{CONCLUSIONES}

Con los resultados obtenidos podemos concluir que adolescentes asmáticos y no asmáticos mostraron baja adherencia a la dieta mediterránea y bajos niveles de actividad física. Los sujetos no asmáticos fueron más activos y tuvieron menor ICT que los sujetos asmáticos, siendo los varones no asmáticos más activos que las mujeres. Por tanto, ambos grupos (asmáticos y no asmáticos) necesitan mejorar sus hábitos alimentarios y niveles de actividad física con especial énfasis en las niñas.

\section{REFERENCIAS BIBLIOGRÁFICAS}

1. A Physical Activity Action [Internet]. Estados Unidos: Designed to Move 2015; [Actualizado 17 Mar 2014; citado 16 Dic 2016]. Disponible en http://es.desingnedtomove.org

2. Gennuso KP, Gangnon RE, Matthews CE, Thraen-Borowski KM, Colbert LH. Sedentary behavior, physical activity, and markers of health in older adults. Med Sci Sports Exerc 2013;45(8):1493-500. https://doi.org/10.1249/MSS.0b013e318288a1e5 PMid:23475142 PMCid:PMC5764165

3. Moral VP. GEMA 4.0. Guía española para el manejo del asma. Arch Bronconeumol 2015;51:2-54 https://doi.org/10.1016/S0300-2896(15)32812-X

4. França-Pinto A, Mendes FA, de Carvalho-Pinto RM, Agondi RC, Cukier A, Stelmach R, et al. Aerobic Training Decreases Bronchial Hyperresponsiveness and Systemic Inflammation in Patients with Moderate or Severe Asthma: A Randomized Controlled Trial. J Emerg Med 2016;3(50):547.

5. Walders-Abramson N, Wamboldt FS, Curran-Everett D, Zhang L. Encouraging physical activity in pediatric asthma: a case-control study of the wonders of walking (WOW) program. Pediatr Pulmonol 2009;44(9):909-16. https://doi.org/10.1002/ppul.21077 PMid:19658109 PMCid:PMC3971919

6. Enfermedades respiratorias crónicas [Internet]. Suiza: Organización Mundial de la Salud. 2016; [Actualizado 20 Mar 2017; citado 7 Abr 2017] Disponivle en http://www.who.int/respiratory/asthma/es/.

7. Grigoropoulou D, Priftis K, Yannakoulia M, Papadimitriou A, Anthracopoulos $\mathrm{M}$, Yfanti $\mathrm{K}$, et al. Urban environment adherence to the Mediterranean diet and prevalence of asthma symptoms among 10-to 12-year-old children: The Physical Activity, Nutrition, and Allergies in Children Examined in Athens study. Allergy Asthma Proc 2011;32(5):351-8. https://doi.org/10.2500/aap.2011.32.3463 PMid:22195687

8. Blasco AJ, Pérez-Yarza EG, Lázaro P, Bonillo A, Díaz CA, Moreno A. Coste del asma en pediatría en España: un modelo de evaluación de costes basado en la 
prevalencia.

An

Pediatr

$2011 ; 74(3): 145-153$.

https://doi.org/10.1016/j.anpedi.2010.10.006 PMid:21339090

9. Saadeh D, Salameh P, Caillaud D, Charpin D, De Blay F, Kopferschmitt C, et al. Prevalence and association of asthma and allergic sensitization with dietary factors in schoolchildren: data from the french six cities study. BMC Public Health 2015;15(1):1. https://doi.org/10.1186/s12889-015-2320-2 PMid:26423141 PMCid:PMC4589972

10. Chen Y, Tu Y, Huang K, Chen P, Chu D, Lee YL. Pathway from central obesity to childhood asthma. Physical fitness and sedentary time are leading factors. Am J Respir Crit Care Med 2014;189(10):1194-203. https://doi.org/10.1164/rccm.20140100970C PMid:24669757

11. Arvaniti F, Priftis KN, Papadimitriou A, Papadopoulos M, Roma E, Kapsokefalou M, et al. Adherence to the Mediterranean type of diet is associated with lower prevalence of asthma symptoms, among 10-12 years old children: the PANACEA study. Pediatr Allergy Immunol 2011;22(3):283-89. https://doi.org/10.1111/j.13993038.2010.01113.x PMid:21457335

12. Moreira A, Delgado L, Haahtela T, Fonseca J, Moreira P, Lopes C, et al. Physical training does not increase allergic inflammation in asthmatic children. Eur Respir J 2008;32(6):1570-75. https://doi.org/10.1183/09031936.00171707 PMid:18684843

13. Basaran S, Guler-Uysal F, Ergen N, Seydaoglu G, Bingol-Karakoc G, Ufuk Altintas D. Effects of physical exercise on quality of life, exercise capacity and pulmonary function in children with asthma. J Rehabil Med 2006;38(2):130-35. https://doi.org/10.1080/16501970500476142 PMid:16546771

14. Onur E, Kabaroğlu C, Günay Ö, Var A, Yilmaz Ö, Dündar P, et al. The beneficial effects of physical exercise on antioxidant status in asthmatic children. Allergol Immunopathol 2011;39(2):90-5. https://doi.org/10.1016/j.aller.2010.04.006 PMid:21242022

15. Serra-Majem L, Ribas L, Ngo J, Ortega RM, García A, Pérez-Rodrigo C, et al. Food, youth and the Mediterranean diet in Spain. Development of KIDMED, Mediterranean Diet Quality Index in children and adolescents. Public Health Nutr 2004;7(07):931-35. https://doi.org/10.1079/PHN2004556 PMid:15482620

16. Aggio D, Fairclough S, Knowles Z, Graves L. Validity and reliability of a modified english version of the physical activity questionnaire for adolescents. Arch Public Health 2016;74:3 https://doi.org/10.1186/s13690-016-0115-2 PMid:26807217 PMCid:PMC4724149

17. Cole TJ, Flegal KM, Nicholls D, Jackson AA. Body mass index cut offs to define thinness in children and adolescents: international survey. BMJ 2007;335(7612):194-197. https://doi.org/10.1136/bmj.39238.399444.55 PMid:17591624 PMCid:PMC1934447

18. Marrodán MD, Martínez-Álvarez JR, De Espinosa MG, López-Ejeda N, Cabañas MD, Prado $C$. Precisión diagnóstica del índice cintura-talla para la identificación del sobrepeso y de la obesidad infantil. Med Clin 2013;140(7):296-301 https://doi.org/10.1016/j.medcli.2012.01.032 PMid:22726769

19. Vargas ME, Souki A, Ruiz G, García D, Mengual E, González CC, et al. Percentiles de circunferencia de cintura en niños y adolescentes del municipio Maracaibo del Estado Zulia, Venezuela. An Venez Nutr 2011;24(1):13-20.

20. García-Marcos L, Martínez A, Batlles J, Morales M, García G, Escribano A. International Study of Asthma and Allergies in Childhood (ISAAC) fase II: metodología y resultados de participación en España. An pediatric 2001; 55:400-5. https://doi.org/10.1016/S1695-4033(01)77711-9

21. Neyra AL, Rodríguez SA, Santiago VS, Fajardo EU, Rivas CT, Asensi JV. Interpretando la función pulmonar. Una perspectiva para el siglo XXI desde la Neumología pediátrica. Rev Patol Respir 2015;18(2). 
22. Quanjer PH, Stanojevic S, Cole TJ, Baur X, Hall GL, Culver BH, et al. Multiethnic reference values for spirometry for the 3-95-yr age range: the global lung function 2012 equations. Eur Respir J 2012;40(6):1324-43. https://doi.org/10.1183/09031936.00080312 PMid:22743675 PMCid:PMC3786581

23. Corder K, Sharp S, Atkin A, Griffin S, Jones A, Ekelund U, et al. Change in objectively measured physical activity during the transition to adolescence. Br J Sports Med 2015;49(11):730-36. https://doi.org/10.1136/bjsports-2013-093190 PMid:24273308 PMCid:PMC4453714

24. Babey SH, Hastert TA, Wolstein J. Adolescent sedentary behaviors: correlates differ for television viewing and computer use. J Adolesc Health 2013;52(1):70-76. https://doi.org/10.1016/j.jadohealth.2012.05.001 PMid:23260837 PMCid:PMC3786734

25. Van Hecke L, Loyen A, Verloigne M, Van der Ploeg, Hidde P, Lakerveld J, Brug $\mathrm{J}$, et al. Variation in population levels of physical activity in European children and adolescents according to cross-European studies: a systematic literature review within DEDIPAC. Int J Behav Nutr Phys Act 2016; 13(1):69-72. https://doi.org/10.1186/s12966016-0395-5 https://doi.org/10.1186/s12966-016-0396-4

26. Rey-López JP, Vicente-Rodríguez G, Biosca M, Moreno LA. Sedentary behaviour and obesity development in children and adolescents. Nutr Metab Cardiovasc Dis 2008;18(3):242-251. https://doi.org/10.1016/j.numecd.2007.07.008 PMid:18083016

27. Arriscado D, Muros JJ, Zabala M, Dalmau JM. Factors associated with low adherence to a Mediterranean diet in healthy children in northern Spain. Appetite 2014;80:28-34. https://doi.org/10.1016/j.appet.2014.04.027 PMid:24798762

28. Gonzalez F, Pertega S, Bamonde L, Garnelo L, Perez Castro T, Sampedro $\mathrm{M}$, et al. Mediterranean diet and asthma in Spanish schoolchildren. Pediatr Allergy Immunol 2010;21(7):1021-27. https://doi.org/10.1111/j.1399 3038.2010.01080.x PMid:20561232

29. Eijkemans M, Mommers M, Jos MT, Thijs C, Prins MH. Physical activity and asthma: a systematic review and meta-analysis. PLoS One 2012;7(12):e50775. https://doi.org/10.1371/journal.pone.0050775 PMid:23284646 PMCid:PMC3527462

30. Rosenkranz SK, Swain KE, Rosenkranz RR, Beckman B, Harms CA. Modifiable lifestyle factors impact airway health in non-asthmatic prepubescent boys but not girls. Pediatr Pulmonol 2011;46(5):464-472. PMid:21194140

Referencias totales / Total references: 30 (100\%) Referencias propias de la revista / Journal's own references: 0

Rev.int.med.cienc.act.fís.deporte - vol. 19 - número 73 - ISSN: 1577-0354 